\title{
Cronobacter species as emerging causes of healthcare-associated infection
}

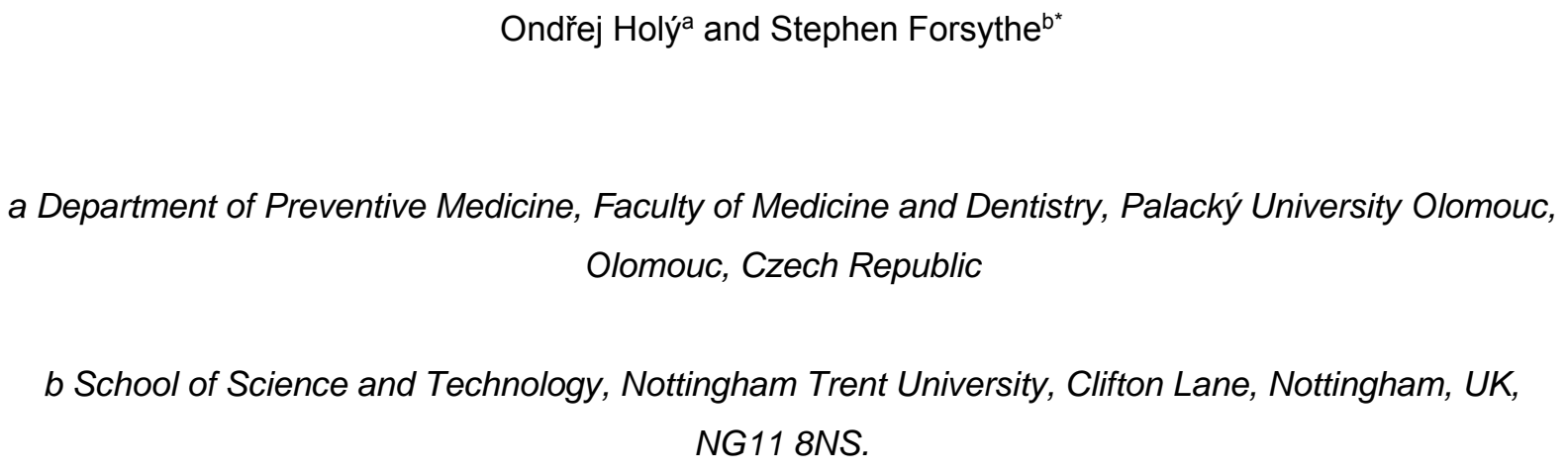




\section{Summary}

\section{Background}

Until recently members of the Cronobacter genus (formerly known as Enterobacter sakazakii) were a relatively unknown cause of hospital infections. However their association with infant infections and in particular through the consumption of contaminated reconstituted infant formula in neonatal intensive care units, has resulted in international efforts to improve neonatal healthcare.

\section{Aim}

This review considers the current status of our understanding of this emergent group of bacterial pathogens and the steps taken to reduce neonatal infection.

Methods

A literature review was undertaken to collate our current knowledge of the Cronobacter genus, with respect to recent taxonomic revisions, sources and clinical relevance.

\section{Findings}

The majority of severe neonatal meningitis infections are associated with only one of the ten Cronobacter species, and in particular the clonal complex known as C. sakazakii ST4. International efforts by FAO-WHO to reduce the risk of neonatal infection by this organism have resulted in improved microbiological safety of powdered infant formula (PIF) and revised guidelines for feeding practices have been problematic. However the majority of infections are in the adult population, the sources of which are unknown.

\section{Conclusion}

International improvements in the microbiological safety of PIF and advice on feeding practices have been directed towards improving neonatal healthcare following the heightened awareness of Cronobacter infections in this particular age group. While these are likely to also reduce neonatal exposure to other opportunistic bacterial pathogens, nevertheless a number of unresolved issues remain with respect to the practicalities of feeding premature neonates safety while following WHO advice. 


\section{General Introduction}

This review concerns the bacterial genus Cronobacter which can cause severe illness in the highly vulnerable neonates, infants and the elderly. The organism has come to prominence due to its association with severe though rare neonatal infections leading to necrotizing enterocolitis, septicaemia and meningitis, which can be fatal. As neonates are frequently fed reconstituted powdered infant formula (PIF), which is not a sterile product, this potential vector has been the focus of attention for reducing infection risk to neonates as the number of exposure routes is limited. Meanwhile, the majority of Cronobacter infections occur in the adult population but are less severe. Cases of Cronobacter infection in all age groups are probably under-reported for a number of reasons such as misidentification as Enterobacter cloacae. ${ }^{1}$ Fortunately our understanding of Cronobacter has grown considerably in recent years. In part this is due to new developments and applications in clinical microbiology of Next Generation Sequencing methods, which have led to rapid improvements in our understanding of this organism; changing our perspective of the former Enterobacter sakazakii species into a genus composed of 10 species, with high clonality and host-adaption, along with improved methods of identification and typing. This review aims to bring together our current knowledge on the clinical aspects of Cronobacter, and consider unresolved issues concerning the hygienic preparation of powdered infant formula.

\section{Cronobacter taxonomy and phylogeny:}

The bacterial genus Cronobacter was formerly known as Enterobacter sakazakii, and was first defined as a new genus in $2007 .^{2}$ It is a member of the Enterobacteriaceae family and is closely related to the Enterobacter and Citrobacter genera. In recent years the Cronobacter genus has undergone a number of revisions and currently contains 10 species. ${ }^{3,4}$ The formally recognised species are $C$. sakazakii, C. malonaticus, C. universalis, C. turicensis, C. muytjensii, C. dublinensis, C. condimenti, C. helveticus, $C$. pulveris and $C$. zurichensis; Figure 1. These include the former bacterial species Enterobacter sakazakii, E. helveticus, E. pulveris and E. turicensis. Subsequently it is uncertain which specific Cronobacter species were referred to in many pre-2007 publications. The species can be grouped, with the mostly clinically relevant being Group1: C. sakazakii and C. malonaticus which form the majority of clinical isolates, and Group 2: C. turicensis and C. universalis which have been less frequently reported. The close relatedness of these species is shown in Figure 1. The other species are primarily environmental commensals and are probably of little clinical significance. According to phylogenetic analysis these major divisions formed about 41 million years ago and further hostadaptation has occurred as will be considered later with the specific case of $C$. sakazakii sequence type 4.5

\section{Cronobacter physiology}

Cronobacter spp. can grow over a wide temperature range. The lowest being near refrigeration temperatures $\left(\sim 5^{\circ} \mathrm{C}\right)$, the optimal $\sim 37-39^{\circ} \mathrm{C}$, with the maximum growth temperature is $44-47^{\circ} \mathrm{C} .{ }^{6}$ The organism's tolerance to desiccation is well recognised. It can survive for two years desiccated in infant formula and then rapidly grow on reconstitution. ${ }^{7}$ The organism often produces a capsule which 
can be so copious that on milk agar plates the colonies drip onto the lid of inverted Petri dishes. ${ }^{8}$ Cronobacter spp. are able to adhere to silicon, latex and polycarbonate, stainless steel, glass and polyvinyl chloride. ${ }^{6,9}$ These materials are commonly used for infant-feeding and food preparation equipment and, if contaminated, may increase the risk of infection. This capsular material may facilitate the organism forming biofilms that are resistant to cleaning and disinfectant agents. ${ }^{10}$ The organism has been isolated as part of the mixed flora biofilm in enteral feeding tubes of neonates. ${ }^{11}$

\section{Virulence traits}

The Cronobacter are opportunistic pathogens though few virulence factors have been identified to date. Some strains can invade human intestinal cells, replicate in macrophages, and invade the blood brain barrier. ${ }^{12}$ Based on the analysis of archived strains and clinical outcome of the 1994 outbreak in France, it was proposed that certain strains of $C$. sakazakii were more virulent, and this has been confirmed by multilocus sequence typing (MLST). ${ }^{13,14}$ The route of infection is probably through attachment and invasion of the intestinal cells, and therefore genes encoding surface appendages such as pili (fimbriae) have been studied. A number of fimbriae clusters were identified in the genomes of Cronobacter species. ${ }^{1,15-17}$ Many are common to all species, though there are some interesting variations. C. sakazakii is the only Cronobacter species encoding for $\beta$-fimbriae, whereas the genomes of the other species encode for curli fimbriae. ${ }^{1,16}$ This may reflect evolution to the host ecosystem.

Since Cronobacter is associated with neonates and infants, the utilization of iron from breast milk and formula could be an important virulence trait. A number of iron assimilation mechanisms have been found in Cronobacter species. ${ }^{1,15-18}$ Type VI secretion system (T6SS) is a newly described system that may be involved in adherence, cytotoxicity, host-cell invasion, growth inside macrophages and survival within the host. Five putative T6SS clusters have been identified Cronobacter spp. genomes. ${ }^{1,17-19}$ It remains to be determined whether they encode functional type VI secretion systems. Cronobacter produce an enterotoxin, and as with neonatal meningitic $E$. coli, the outer membrane proteins ompA and ompX possibly have roles in the organism penetrating the blood brain barrier. The mechanism(s) leading to the destruction of the brain cells in unknown and could in part be a host response. The organism also encodes for a number of haemolysins. ${ }^{1,19}$

C. sakazakii is unique in the Cronobacter genus in its utilization of exogenous sialic acid, and this may have clinical significance. The ability to utilise sialic acid could be a major evolutionary hostadaptation since the compound is found in breast milk, mucin and gangliosides. ${ }^{20}$ Sialic acid is also an ingredient in powdered infant formula due to its association with brain development. $C$. sakazakii is also able to grow on the ganglioside GM1 as a sole carbon source. ${ }^{20}$

High levels of heat-stable lipopolysaccharide (LPS, also known as endotoxin) in infant formula enhances the translocation of Cronobacter across both the intestines and the blood-brain barrier, and therefore increase the risk of bacteraemia in neonates. ${ }^{23,24,25} \mathrm{Kim}$ and Loessner speculated that frequent LPS contamination of PIF (known to disrupt tight junctions) might contribute to the invasiveness of Cronobacter across the blood-brain barrier. ${ }^{24}$ It is known that the levels of LPS in PIF 
vary 500 -fold. ${ }^{25}$ In addition, the oligopolysaccharide component of the LPS layer can serve as a basis for serotyping and other characterisation methods. ${ }^{21,22}$

Cronobacter spp. tend to be more sensitive to most antibiotics compared to other Enterobacteriaceae, though resistance to ampicillin has developed. In 1980, all strains tested were susceptible to ampicillin, whereas in 2001 Lai described five cases of Cronobacter infection in which one or more of the isolates were resistant to ampicillin and most cephalosporins of $1^{\text {st }}$ and $2^{\text {nd }}$ generation. ${ }^{26,27}$ In 2001, Lai reported increasing $\beta$-lactamase production among Cronobacter strains. ${ }^{27}$ Similarly, Block and colleagues reported that all Cronobacter isolates tested were $\beta$-lactamase positive. ${ }^{28}$ Caubilla-Barron et al. in a retrospective study of a NICU outbreak in 1994, reported two neonatal deaths from ESBL-encoding C. sakazakii strains. ${ }^{8}$ It is of interest that indistinguishable pulsetype strains were non-ESBL, indicating the possible acquisition of the ESBL genes from the individual neonatal intestinal flora during the infection period.

\section{Non-human sources of Cronobacter spp.}

Cronobacter spp. has been isolated from a range of foods including cheese, meats, milk powder, powdered infant formula, weaning foods, and a large portion of food ingredients; Table 1. ${ }^{29,30}$ Although the bacterium is isolated from many foods, no foodborne infections have not been reported. A productive source of Cronobacter strains are fresh or dried herbs and spices with $\sim 30 \%$ incidence ${ }^{29}$. Rats, flies and cockroaches may be additional sources of contamination. ${ }^{31,32,33}$

The bacterium has been isolated from the home environment; household dust, vacuum cleaning bags and also from utensils used for the reconstitution of powdered infant formula; Table $1 .{ }^{34}$ Hence contamination of reconstituted infant formula can be intrinsic or extrinsic in origin. The bacterium has also been isolated hospital environments, as well as various areas in milk powder and PIF processing plants; roof, shoes, and roller driers. ${ }^{35,36,37,38}$

The organism has been recovered from previously unopened tins of powdered infant formula indicating intrinsic contamination; Table $1 .{ }^{39,40,41}$ Accurate enumeration is difficult but is generally in the order of $<1$ bacterial cells $/ 100 \mathrm{~g}$. The intrinsic prevalence of Cronobacter in powdered infant formula has been determined a number of times and varies between $2-14 \%$, with no published reports exceeding $1 \mathrm{cell} / \mathrm{g}$. Hence the consideration of opportunities for extrinsic bacterial contamination, and multiplication, especially temperature abuse following reconstitution as it would allow the bacterium to multiply and increase the risk of infection. Currently microbiological criteria for Cronobacter spp. are required for infant formulas with an intended target age $<6$ months..$^{42} \mathrm{~A}$ presence/absence test is applied to large volumes due to the low incidence of the organism in the product. Although the organism has been recovered from follow up formulas (infant formulas with intended target age $>6$ months) and weaning foods, there is currently insufficient epidemiological evidence to support the implementation of criteria for these products.

A limited number of studies have shown that Cronobacter is waterborne. ${ }^{3,43,44}$ This important issue has not received as much attention as bacterial contamination of PIF. As will be considered later, since powdered infant formula is not a sterile product one method of reducing neonatal exposure to Cronobacter has been the recommendation to reconstitute with water $>70^{\circ} \mathrm{C}$ to kill any vegetative 
bacteria present. ${ }^{39,40,45}$ This advice has not been adopted by all countries. It should be noted that detailed microbiological examination of the USA December 2011 infant infection cases revealed the presence of $C$. sakazakii in the PIF reconstitution water which was a close match (differed by 35/3036 nucleotides of the MLST alleles) to that recovered from the cerebral spinal fluid of the associated infant with meningitis. ${ }^{14}$

\section{Human and clinical sources of Cronobacter spp.}

Asymptomatic human carriage of Cronobacter spp. has been reported in a few studies with recovery from mouth, skin and faeces; Table 1.

Cronobacter spp. have been isolated from various hospital environmental and clinical samples; cerebrospinal fluid, blood, bone marrow, sputum, urine, inflamed appendix, breast abscess, and conjunctivae. ${ }^{3,5,8,13,16,27,46,47}$ Nazarowec-White and Farber studying three isolates obtained from one hospital over 11 years showed that they were indistinguishable. ${ }^{48}$ Smeets et al. showed that isolates from a contaminated dish brush used for cleaning bottles in a hospital and the isolates from three patients were identical making an epidemiological connection likely. ${ }^{49}$ The organism has also been isolated from a doctor's stethoscope and from nursery food preparation equipment such as spoons and a blender. ${ }^{26,50,51,52}$ The organism has been found as part of the mixed flora biofilm in enteral feeding tubes of neonates not fed PIF. ${ }^{11}$ Related to this, laboratory studies have shown that one contaminated feed passing through the feeding tube would subsequently contaminate further feeds due to bacterial attachment to the inner tube wall and multiplication. ${ }^{53}$

\section{Isolation, identification and typing methods}

As the organism has only been reported at low numbers ( $<1 \mathrm{cfu} / \mathrm{g}$ ) in PIF, a large volume of material needs to be tested in microbiological analysis. Therefore presence/absence testing of bacteria in PIF is applied rather than direct enumeration. This includes the use of chromogenic agars, along with DNA-based identification and fingerprinting techniques. Although it is generally possible to differentiate Cronobacter species by biochemical profiling, molecular methods are increasingly used as a more rapid and reliable tool to study bacterial genomic diversity and to track sources of infection. Since the organism is ubiquitous, typing schemes are required both for epidemiological and environmental investigation. For epidemiological analysis (ie. tracing source and dissemination during an outbreak), PFGE with two restriction enzymes (Xba1 and Spe1) is the most common method. ${ }^{8}$ The technique is widely employed and can be used for transnational investigations, as per PulseNet, since the gel results can be electronically analyzed (http://www.cdc.gov/pulsenet/). The method is limited however as not all strains can be typed, non-identical strains can give the same PFGE profile and the method does not give the relationship between strains. ${ }^{35,54}$

On a larger scale, multilocus sequence typing (MLST) is increasingly being applied to understand the evolution and diversity of bacterial pathogens, for example E. coli ST131, MRSA-15 ST22 and Klebsiella pneumoniae ST258. ${ }^{55}$ The method defines sequence types (ST) based on 7 allelic profiles and clonal complexes based on relatedness of the sequence types (1-3 loci differences). The MLST scheme for Cronobacter has been established and is available online with approximately 600 strains 
profiles (www.pubMLST.org/cronobacter/) ${ }^{5,56,57}$ The site also includes open access for the further analysis of all published Cronobacter genome sequences using the 'Bacterial Isolate Genomes Sequence Database' (BIGSdb) facility. ${ }^{58}$ The web site contains the MLST protocols, as well as $>200$ DNA sequence defined profiles for strains which have been collected from various sources and countries over a 60 year period. The Cronobacter MLST analysis is based on 7 housekeeping genes; atpD, fusA, glnS, gltB, gyrB, infB and ppsA. The 7 sequenced alleles can be concatenated together to give 3036 nucleotide sequence for phylogenetic analysis (Fig 1). This analysis has revealed a remarkably strong clonal nature in the Cronobacter genus. ${ }^{5,57}$ These clones may reflect different ecologies of the organism. The clonal complex C. sakazakii ST4 is a DNA sequence defined evolutionary lineage for the causative agent of neonatal meningitis among the Cronobacter isolates. $5,13,14,57$ This remarkable discovery gives a clear direction for further meningitis research with the bacterium. Neisseria meningitidis also shows clonality of meningitis infection. To date there does not appear to be such a clear link between sequence type and other Cronobacter infections, such as necrotizing enterocolitis.

It should be noted that $16 \mathrm{~S}$ rDNA sequence analysis of archived strains has revealed that the use of phenotyping in early studies led to a number of mis-identifications in the literature. ${ }^{16}$ These include:

1. An independent fatal case of neonatal sepsis due to E. cloacae during a C. sakazakii outbreak in a neonatal intensive care unit. $^{8}$

2. Neonatal intensive care unit outbreak attributed to $E$. sakazakii, reidentified as $E$. hormaechei. ${ }^{59}$

3. A reported quinolone-resistant E. sakazakii strain, reidentified as $E$. hormaechei. ${ }^{60}$

4. E. sakazakii strain used for oligo-polysaccharide structure determination, re-identified as $E$. ludwiggi. ${ }^{61}$

Such mis-identifications are likely to continue given that the database for a commonly used phenotyping based method does not recognised the 2007 taxonomic revision of Cronobacter and continues to use the old $E$. sakazakii nomenclature, and three of the most recently defined Cronobacter species (C. helveticus, C. pulveris and C. zurichensis) are not recognised even as $E$. sakazakii.

\section{Cronobacter spp. infections}

A number of Cronobacter infection incidents have been reported as outbreaks. ${ }^{8,39,40}$ In the USA, the reported Cronobacter infection incidence rate is 1 per 100000 infants. This incidence rate increases to 9.4 per 100000 in infants of very low birth weight, i.e. $<1.5 \mathrm{~kg} .{ }^{40}$ Fatal Cronobacter infections of infants have followed cases of necrotising enterocolitis (NEC), septicaemia and meningitis. ${ }^{62,63}$ Infections in older age groups are principally bacteraemia as well as urosepsis and wound infections. Infants can be colonized by more than one strain of Cronobacter, and therefore multiple isolates need to be characterized in epidemiological investigations. ${ }^{8}$

NEC is non-invasive (as well as multifactorial), whereas in septicaemia and meningitis the organism has attached and invaded presumably through the intestinal epithelial layer. NEC is a common gastrointestinal illness in neonates and can be caused by a variety of bacterial pathogens. It 
is characterized by ischaemia, bacterial colonisation of the intestinal tract, and increased levels of proteins in the gastrointestinal lumen. The incidence of NEC is $2-5 \%$ of premature infants and $13 \%$ in those weighing $<1.5 \mathrm{~kg}$ at birth. It is 10 times more common in infants fed formula compared with those fed breast milk. NEC has a high mortality rate; $15-25 \%$ of cases. Cronobacter has been implicated as a causative agent of NEC, but its role in the pathogenesis of the disease is somewhat unclear. There are reports of Cronobacter isolation from babies who developed NEC and these strains were indistinguishable by PFGE from those isolated from meningitis cases. ${ }^{8}$ This suggests that there is an association between Cronobacter occurrence and NEC, although until recently, the organism has not been conclusively proven to cause the disease.

Cronobacter-related meningitis is characterized by a mortality rate of $40-80 \%$ and generally a very poor clinical outcome. The bacterium causes cystic changes, abscesses, fluid collection, brain infarctions, hydrocephalus, necrosis of brain tissue and liquefaction of white cerebral matter. This pathogenesis is different to that caused by both Neisseria meningitidis and neonatal meningitic E. coli. Some reports suggest a similarity between the tropism of Cronobacter and the closely related organism Citrobacter koseri for invasion and infection of the central nervous system. Patients surviving Cronobacter-related meningitis often suffer from severe neurological sequelae, such as hydrocephalus, quadriplegia and retarded neural development. ${ }^{16,62,63}$ The infection usually arises between the fourth and fifth day after birth and it can be fatal within hours to days following the first clinical signs. Compared with patients suffering from Cronobacter-induced enterocolitis, infants in whom meningitis developed tend to have normal gestational age and birth weight. In December 2011, there was considerable publicity concerning neonatal Cronobacter infections in the United States. ${ }^{64}$ All but one isolate from the meningitic cases were in the $C$. sakazakii ST4 clonal complex. $^{14}$

Infections caused by Cronobacter in adults comprise a wide range of symptoms from conjunctivitis, biliary sepsis, urosepsis and appendicitis to wound infection and pneumonia. Adult patients at increased risk include those previously treated with antibiotics, immuno-compromised and elderly patients, those with medical implants or with acute, chronic, or serious illnesses. C. sakazakii can cause urinary tract infections, though to date this aspect has not been studied in any detail. The only published age-profiled data is for 819 Cronobacter spp. bacteraemia cases reported for England and Wales between 1992 and $2007 .{ }^{41}$ In this report, the majority (91\%) of bacteraemia cases were patients $>15$ years in age.

\section{Sources of infection}

While the source of contamination in Cronobacter-related outbreaks has not always been confirmed, breast milk substitutes (one group of PIF products) have been epidemiologically or microbiologically

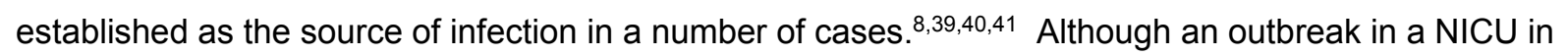
Tennessee in 2001 is often cited as a strong link between the presence of Cronobacter in powdered infant formula and Cronobacter infection, it is overlooked that the formula fed to the infant in Tennessee was in fact a non-infant formula and was not intended to be consumed by neonates. ${ }^{65}$ 
As already covered, the $C$. sakazakii clonal complex ST4 is strongly associated with cases of meningitis and it is notable that this clonal complex has been reported to be frequently isolated from milk powder factories, powdered infant formula (PIF) processing plants and from PIF in Ireland, Switzerland, Germany and Australia. ${ }^{36,37,38}$ Sonbol et al. reported that $24 \%$ of strains isolates from the environment of 6 milk powder manufacturing plants in Australia and Germany were C. sakazakii ST4. ${ }^{38}$

Infections which have been directly linked to reconstituted PIF may have been the result of intrinsic or extrinsic contamination during preparation and administration. A common feature in some of these outbreaks is the opportunity for temperature abuse of the prepared feed, which would permit bacterial growth. In reported outbreaks in France and USA, the neonates were fed using perfusion devices where the reconstituted PIF is slowly pumped over several hours at ambient temperature into the neonate's stomach through an enteral feeding tube. ${ }^{8,65}$ Using this procedure there is the possibility of bacterial multiplication in the syringe leading to the ingestion of large numbers of Cronobacter by the neonate.

The neonate has an immature immune system and a low intestinal microflora density. Consequently, if a large number of Cronobacter cells were ingested they would not be outcompeted by the resident intestinal flora. Following invasion of the intestinal cells, the lack of a developed immune system could make the neonate more prone to systemic infection. No infectious dose has been determined for neonates. Animal studies by Pagotto and others have used large numbers of Cronobacter cells $\left(\sim 10^{8}\right)$ for infection studies. ${ }^{66}$ Whether this number is reflective of that necessary for neonate infection is uncertain, but it does contrast with the number of cells reported in contaminated PIF ( $<1 \mathrm{cfu} / \mathrm{g}$ ), and may therefore indicate the significant role of temperature abuse in enabling bacterial multiplication.

It is pertinent to note that Cronobacter sakazakii has been isolated from the tracheae, and sputum as well as from the feeding tubes of neonates fed breast milk and ready-to-feed formula, not infant formula; Table 1.8,11,67 Therefore wider sources of the organism during an outbreak need to be investigated, not just the use of PIF. The 1994 NICU outbreak in France showed that infants can be colonized by more than one strain of Cronobacter, and therefore multiple isolates need to be genotyped in epidemiological investigations to increase the chance of tracing probable source. ${ }^{8}$

Bowen and Bradden reported that there are a number of neonatal cases which have no links with the ingestion of reconstituted infant formula. ${ }^{63}$ Pertinent to this, is the recognition of bacterial colonisation of the nasogastric enteral feeding tube, and the isolation of $C$. sakazakii from tubes from neonates feed breast milk, and (sterile) ready to feed formula. Breast milk can contain the bacterium, and the C. malonaticus type strain (LMG $\left.23826^{\top}\right)$ was isolated from a breast abscess. In some countries breast milk from mothers with mastitis is still fed to the neonate. Breast milk has been a suspect source in two meningitis cases. ${ }^{68,69}$ Cronobacter infections of babies with breast milk as sole source of feed have been reported in US and Israel; Block pers. comm..$^{28,70}$

Cronobacter species have also been isolated from hospital air, dust, human intestines and throats. Hence control of microbiological content of PIF will not necessarily totally remove the risk of neonate infection by this bacterium. 


\section{Consequences and current issues.}

To date the raised awareness of the organism has focussed on infant infections and resulted in changes in the microbiological criteria for PIF and reconstitution procedures. ${ }^{39,40,41,42}$ Those identified as being at high risk of Cronobacter infection are neonates (especially low birth weight) for whom their source of nutrition will be limited to breast milk, fortified breast milk, or breast milk replacement. Hygienic preparation of feed is essential due to their immature immune system and lack of competing intestinal flora. Key advice from these FAO-WHO risk assessments was that PIF should be reconstituted with water $>70^{\circ} \mathrm{C}$, minimise any storage time by not preparing in advance and if storage for short periods is necessary then the temperature should be $<5^{\circ} \mathrm{C}$. The high water temperature will drastically reduce the number of vegetative bacteria present, and minimising the storage period will reduce the multiplication of any surviving organisms. These recommendations have been well addressed by the WHO 'Guidelines for the safe preparation, storage and handling of powdered infant formula'. ${ }^{42}$ However this has subsequently caused a number of additional issues for weaning foods, follow-on formulas, fortified breast milk, and probiotic-supplemented PIFs as consider below.

As referred to above, the WHO guidelines for hygienic preparation of PIF are aimed at reducing the number of bacteria in the reconstituted product by using hot water and limiting the time available for any survivors to multiply. ${ }^{42}$ However a wider perspective is that neonates are frequently feed via enteral feeding tubes. These tubes are in place for prolonged periods (even several days) to reduce distress to the neonate by the gagging reaction. However Cronobacter, and other opportunistic pathogens can attach and colonise these tubes which are at $37^{\circ} \mathrm{C}$, and at regular intervals receive fresh feed. ${ }^{11}$ This scenario is applicable to all neonates with nasogastric tubes, and not only those on reconstituted PIF. In fact Cronobacter and other Enterobacteriaceae have been isolated from such tubes in intensive care units from neonates receiving breast milk and various other feeding regimes at levels up to $10^{7}$ cfu per tube. ${ }^{11}$ Therefore hygienic practices and avoidance of temperature abuse are vitally important regardless of the type of feed.

\section{So where are we now?}

1. Ironically despite the international changes, the original Tennessee outbreak which precipitated the FAO-WHO risk assessments and WHO preparation of PIF guidelines could still occur. Why? Because the outbreak was due to the use of formula that was not intended for consumption by infants, therefore the product is currently not subject to the revised microbiological criteria.

2. The FAO-WHO recommended reconstitution of powdered infant formula with water $>70^{\circ} \mathrm{C}$ is not followed in all countries such as the US, although it is supported by the CDC. Dipping a thermometer into reconstituted formula would have its own inherent problems of contamination and so the advice has been to use water which has been boiled in a kettle and left to cool for 30 minutes. Aside from the variation in cooling curves according to volume of water and type of kettle, this is impractical for premature babies who require only small volumes of formula, and are fed at 2 hourly intervals. This practice may result in staff being 
taken away from bedside care to oversee feed preparation. The term 'powdered infant formula' includes 'breast milk fortifiers'. These products are added to supplement the nutritional value of mother's milk. They are not reconstituted with water and cannot receive the heat-treatment to kill intrinsic bacteria. The use of high-temperature for reconstitution precludes the inclusion of probiotic bacterial cultures (such as Lactobacillus fermentum and $L$. reuteri) in PIFs, as marketed in some countries.

3. Most Cronobacter infections are in adults, possibly primarily due C. malonaticus. ${ }^{5,16}$ The source of infection maybe through ingestion as the organism is ubiquitous in food, however it is also plausible that it is nasopharyngeal (like Neisseria meningitis) which could explain the cases of pneumonia and isolation from sputum.

4. The source of $C$. sakazakii ST4 is of considerable interest since controlling this lineage could reduce neonatal exposure to severe, life-threatening infections. An informed assessment of neonatal exposure warrants further investigation for the prevalence of Cronobacter spp., especially ST4, in hospitals, PIF and other sources such as human carriage. ${ }^{47}$

Late onset Gram negative bacterial sepsis remains a significant cause of neonatal morbidity/mortality and infections on NICUs are predominantly due to Enterobacteriaceae, whereas non-fermenting bacteria (ie. Pseudomonas spp.) predominate in other ICU outbreaks. ${ }^{71,72}$ A recent UK neonatal unit outbreak has lead to development of specific national guidelines (under consultation) on the prevention and management of Gram negative sepsis in neonates. ${ }^{73}$ The incidence of sepsis in premature babies in England is 8/100 live births, and 71/1000 neonatal admissions. ${ }^{74}$ It should be noted that Cronobacter spp. are not the only Enterobacteriaceae isolated from PIF, and that the FAOWHO recommended that research should be undertaken into the other Enterobacteriaceae and Acinetobacter spp. in PIF. ${ }^{39,40}$ These organisms were termed 'Category B; plausible causing infections, but without supporting epidemiological evidence' by the expert committees, whereas Cronobacter spp. and Salmonella serovars were 'Category A: Clear evidence of causality'. A wide range of Enterobacteriaceae can be present in PIF, and are the same species as occur in neonatal infections. However cases linking isolates from infants and PIF have not be substantiated. . $^{8,59,75}$

Therefore, despite considerable improvement in our understanding of the emergent bacterial pathogen now known as Cronobacter, there remains a number of practical issues concerning the hygiene feeding of neonates, and the possible significance in adult infections which are not studied to date.

\section{Acknowledgements}

This work was supported by Research Support Foundation, Vaduz [801100021/39] and Nottingham Trent University.

\section{References}

1. Joseph S, Desai $P$, Ji $Y$, Cummings $C A$, et al. Comparative analysis of genome sequences covering the seven Cronobacter species. PLoS ONE 2012;7:e49455. 
2. Iversen C, Lehner A, Mullane N, et al. The taxonomy of Enterobacter sakazakii: proposal of a new genus Cronobacter gen. nov. and descriptions of Cronobacter sakazakii comb. nov. Cronobacter sakazakii subsp. sakazakii, comb. nov., Cronobacter sakazakii subsp. malonaticus subsp. nov., Cronobacter turicensis sp. nov., Cronobacter muytjensii sp.nov., Cronobacter dublinensis sp. nov. and Cronobacter genomospecies 1. BMC Evol Biol 2007;7:64.

3. Joseph S, Cetinkaya E, Drahovska H, Levican A, Figueras M, Forsythe SJ. Cronobacter condimenti sp. nov., isolated from spiced meat and Cronobacter universalis sp. nov., a novel species designation for Cronobacter sp. genomospecies 1, recovered from a leg infection, water and food ingredients. Intl J System Evol Microbiol 2012; 62:1277-1283.

4. Brady C, Cleenwerck I, Venter S, Coutinho T, De Vos P. Taxonomic evaluation of the genus Enterobacter based on multilocus sequence analysis (MLSA). Syst Appl Microbiol 2013;36: 309-319.

5. Joseph S, Sonbol H, Hariri S, Desai P, McClelland M, Forsythe SJ. Diversity of the Cronobacter genus as revealed by multi locus sequence typing. J Clin Microbiol 2012;50: 3031-3039.

6. Iversen C, Lane M, Forsythe, SJ. The growth profile, thermotolerance and biofilm formation of Enterobacter sakazakii grown in infant formula milk. Lett Appl Microbiol 2004; 38:378-382.

7. Caubilla-Barron J, Forsythe, S. Dry stress and survival time of Enterobacter sakazakii and other Enterobacteriaceae. J Food Protect 2007;70:2111-2117.

8. Caubilla-Barron J, Hurrell E, Townsend S, et al. Genotypic and phenotypic analysis of Enterobacter sakazakii strains from an outbreak resulting in fatalities in a neonatal intensive care unit in France. J Clin Microbiol 2007;45:3979-3985.

9. Lehner A, Riedel K, Eberl L, Breeuwer P, Diep B, Stephan R. Biofilm formation, extracellular polysaccharide production, and cell-to-cell signaling in various Enterobacter sakazakii strains: aspects promoting environmental persistence. J Food Protect 2005;68:2287-94.

10. Beuchat LR, Kim H, Gurtler JB, Lin LC, Ryu JH, Richards GM. Cronobacter sakazakii in foods and factors affecting its survival, growth, and inactivation. Intl J Food Microbiol 2009;136:204213.

11. Hurrell E, Kucerova $E$, Loughlin $M$, et al. Neonatal enteral feeding tubes as loci for colonisation by members of the Enterobacteriaceae. BMC Infec Dis 2009;9:146.

12. Townsend SM, Hurrell E, Gonzalez-Gomez I, et al. Enterobacter sakazakii invades brain capillary endothelial cells, persists in human macrophages influencing cytokine secretion and induces severe brain pathology in the neonatal rat. Microbiology 2007;153:3538-3547.

13. Joseph S, Forsythe SJ. Predominance of Cronobacter sakazakii sequence type 4 in neonatal infections. Emerg Infect Dis 2011;17:1713-1715.

14. Hariri S, Joseph S, Forsythe SJ. Cronobacter sakazakii ST4 strains and neonatal meningitis, United States. Emerg Infect Dis 2013;19:175-177.

15. Kucerova E, Clifton SW, Xia XQ, et al. Genome sequence of Cronobacter sakazakii BAA-894 and Comparative Genomic Hybridization analysis with other Cronobacter species. PLoS ONE 2010;5:e9556.

16. Kucerova E, Joseph S, Forsythe S. Cronobacter: diversity and ubiquity. Qual Ass Safety Foods Crops 2011;3:104-122.

17. Grim CJ, Kotewicz ML, Power KA, et al. Pan-genome analysis of the emerging foodborne pathogen Cronobacter spp. suggests a species-level bidirectional divergence driven by niche adaptation. BMC Genomics 2013;14:366

18. Grim CJ, Kothary MH, Gopinath G, et al. Identification and characterization of Cronobacter iron acquisition systems. Appl Environ Microbiol 2012;78:6035-6050.

19. Cruz A, Xicohtencatl-Cortes J, Gonzalez-Pedrajo B, Bobadilla M, Eslava C, Rosas I. Virulence traits in Cronobacter species isolated from different sources. Can J Microbiol 2011;57:735744.

20. Joseph S, Hariri S, Masood N, Forsythe S. Sialic acid utilization by Cronobacter sakazakii. Microbial Informatics Experiment 2013;3:3.

21. Jarvis KG, Grim CJ, Franco AA, et al. Molecular characterization of Cronobacter lipopolysaccharide O-antigen gene clusters and development of serotype-specific PCR assays. Appl Environ Microbiol 2011;77:4017-4026.

22. Sun Y, Wang M, Wang Q, et al. Genetic analysis of the Cronobacter sakazakii O4 to O7 Oantigen gene clusters and development of a PCR assay for identification of all C. sakazakii O serotypes. Appl Environ Microbiol 2012:78;3966-3974. 
23. Townsend S, Hurrell E, Forsythe S. Virulence studies of Enterobacter sakazakii isolates associated with a neonatal intensive care unit outbreak. BMC Microbiol 2008;8:64.

24. Kim KP, Loessner MJ. Enterobacter sakazakii invasion in human intestinal Caco-2 cells requires the host cell cytoskeleton and is enhanced by disruption of tight junction. Infect Immun 2008;76:562-570.

25. Townsend S, Caubilla-Barron J, Loc-Carrillo C, Forsythe S. The presence of endotoxin in powdered infant formula milk and the influence of endotoxin and Enterobacter sakazakii on bacterial translocation in the infant rat. Food Microbiol 2007;24:67-74.

26. Farmer JJ, Asbury MA, Hickman FW, Brenner DJ, Enterobacteriaceae Study Group (USA). Enterobacter sakazakii: a new species of "Enterobacteriaceae" isolated from clinical specimens. Intl J Syst Bacteriol 1980;30:569-584.

27. Iversen C, Forsythe SJ. Isolation of Enterobacter sakazakii and other Enterobacteriaceae from powdered infant formula milk and related products. Food Microbiol 2004; 21 : 771-776.

28. Lai KK. Enterobacter sakazakii infections among neonates, infants, children, and adults. Case reports and a review of the literature. Medicine 2001;80:113-122.

29. Kim K, Jang SS, Kim SK, Park JH, Heu S, Ryu S. Prevalence and genetic diversity of Enterobacter sakazakii in ingredients of infant foods. Intl J Food Microbiol 2008;122:196-203.

30. Block C, Peleg O, Minster N, et al. Cluster of neonatal infections in Jerusalem due to unusual biochemical variant of Enterobacter sakazakii. Euro J Clin Microbiol Infect Dis 2002;21:613616.

31. Chap J, Jackson P, Siqueira R, et al. International survey of Cronobacter sakazakii and other Cronobacter spp. in follow up formulas and infant foods. Intl J Food Microbiol 2009;136:185188.

32. Hamilton JV, Lehane MJ, Braig HR. Isolation of Enterobacter sakazakii from midgut of Stomoxys calcitrans. Emerg Infect Dis 2003;9:1355-1356.

33. Pava-Ripoll M, Pearson RE, Miller AK, Ziobro GC. Prevalence and relative risk of Cronobacter spp., Salmonella spp., and Listeria monocytogenes associated with the body surfaces and guts of individual filth flies. Appl Environ Microbiol 2012;78:7891-7902.

34. García F, Notario MJ, Cabanás JM, Jordano R, Medina LM. Incidence of bacteria of public health interest carried by cockroaches in different food-related environments. J Med Entomol 2012; 49:1481-1484.

35. Craven HM, McAuley CM, Duffy LL, Fegan N. Distribution, prevalence and persistence of Cronobacter (Enterobacter sakazakii) in the nonprocessing and processing environments of five milk powder factories. J Appl Microbiol 2010;109:1044-1052.

36. Power KA, Yan Q, Fox EM, Cooney S, Fanning S. Genome sequence of Cronobacter sakazakii SP291, a persistent thermotolerant isolate derived from a factory producing powdered infant formula. Genome Announc 2013;1:e0008213

37. Müller A, Stephan R, Fricker-Feer C, Lehner A. Genetic diversity of Cronobacter sakazakii isolates collected from a Swiss infant formula production facility. J Food Prot 2013;76:883887.

38. Sonbol H, Joseph S, McAuley C, Craven H, Forsythe SJ. Multilocus sequence typing of Cronobacter spp. from powdered infant formula and milk powder production factories. Intl Dairy J 2013;30:1-7.

39. FAO-WHO. Workshop on Enterobacter sakazakii and other microorganisms in powdered infant formula. Geneva, Switzerland. 2004.

40. FAO-WHO. Expert meeting on Enterobacter sakazakii and Salmonella in powdered infant formula. Rome, Italy. 2006.

41. FAO-WHO. Enterobacter sakazakii (Cronobacter spp.) in powdered follow-up formulae. Microbiological Risk Assessment Series No. 15. Washington, USA. 2008.

42. Codex Alimentarius Commission (CAC). Code of hygienic practice for powdered formulae for infants and young children. CAC/RCP 66-2008.

43. Lee DG, Kim SJ. Bacterial species in biofilm cultivated from the end of the Seoul water distribution system. J Appl Microbiol 2003;95:317-324.

44. Liu H, Yang Y, Cui J, et al. Evaluation and implementation of a membrane filter method for Cronobacter detection in drinking water. FEMS Microbiol Lett 2013;344:60-68.

45. World Health Organisation. Guidelines for the safe preparation, storage and handling of powdered infant formula. 2007.

46. Palcich G, Gillio Cde M, Aragon-Alegro LC, et al. Enterobacter sakazakii in dried infant formulas and milk kitchens of maternity wards in São Paulo, Brazil. J Food Prot 2009;72:3742. 